\title{
A Review on aptamer-conjugated quantum dot nanosystems for cancer imaging and theranostic
}

\begin{abstract}
Over the last 10 years, fluorescent semiconductor QD (quantum dot)-aptamer conjugates have emerged as an efficient platform for cancer imaging and therapy in animal models and in vitro. In addition, these conjugates show potential in a wide range of applications in environmental monitoring, disease diagnosis, and bio-sensing. The present review represents the recent developments in QD-aptamer bio-conjugates for applications in cancer studies. It starts with a brief introduction to Semiconductor Quantum dots (QDs), bio-conjugation of QDs and aptamer molecules, and advantages-disadvantages of using these novel tools for biochemical applications.
\end{abstract}

Keywords: Theranostic, Aptamer, Cancer, Quantum dot, Imaging
Volume 5 Issue 3 - 2017

\author{
Elmira Samimi,' Pari Karami, ${ }^{2}$ Mohammad \\ Johari Ahar ${ }^{3}$ \\ 'Tabriz University of medical Sciences, Iran \\ 2Department of Analytical Chemistry, University of Tabriz, Iran \\ ${ }^{3}$ Department of Medicinal Chemistry and drug discovery, \\ Ardabil University of Medical Sciences, Iran
}

\begin{abstract}
Correspondence: Mohammad Johari Ahar, Department of Medicinal Chemistry and drug discovery, Faculty of Pharmacy, Ardabil University of Medical Sciences, Iran, Tel 98-45-3352243739, Fax 98-45-33522197 Email johariahar@gmail.com
\end{abstract}

Received: October 03, 2017 | Published: April 18, 2017

Abbreviations: PET, Positron Emission Tomography; MRI, Magnetic Resonance Imaging; CT, Computed Tomography; QD, Quantum Dot

\section{Introduction}

Cancer diagnosis and therapy remains a major obstacle worldwide. An increasing rate of cancer mortality is expected to growth to about 13 million deaths per year by $2030 .{ }^{1}$ Currently, six powerful diagnostic modalities including computed tomography (CT), magnetic resonance imaging (MRI), positron emission tomography (PET-scan), single-photon emission CT, ultrasound and optical imaging are available for cancer imaging and staging .2 However, classifying the cancers and delivering proper dosages with maximum therapeutic effects and minimum toxicity of therapeutics are the main hurdles that seems clinicians have to overcome in the field of cancer treatment. In addition, immune-editing process supporting cancer cells in escaping from the human immune system help tumor cells survive and metastasize other organs . ${ }^{3}$

In recent two decades, nanotechnology as an emerging scientific discipline has received significant attention in all areas of science encompassing chemistry, physics and biology ${ }^{4}$ Indeed, nanotechnology is ever more firming a foothold in medicine, especially in oncology .5 Nanoparticles such as quantum dots and superparamagnetic iron oxide (SPIO) are useful contrast agents for medical imaging with CT or MRI in animal studies . ${ }^{6}$ Among quantum dots, fluorescent inorganic quantum dots (QDs) with diameter of 2 to $10 \mathrm{~nm}$ display superior fluorescent properties without photobleaching in comparing with organic fluorophores.$^{7}$ Significant evidences suggest that QDs can be used in imaging.$^{8}$ It seems that one potential area of application would be in the development of advanced materials for early diagnosis or convergence of imaging and therapy (theranostic) of cancer.

\section{Fluorescent Semiconductor Quantum dots (QDs)}

QDs are nanocrystals whose size-tunable fluorescence can cover electromagnetic spectrum generally from the ultraviolet (UV) to the near infrared (NIR) regions . ${ }^{9}$ As a rule, QDs with a large diameter emit fluorescence in red region, whilst QDs with a smaller diameter radiate photons in the blue region.$^{10}$ Narrow and symmetric emission peaks obtained by excitation with broad ranges of wavelengths render multicolored single QD that enables multimodal cancer imaging ." For this reason, these QDs can be suggested as a potential candidate multimodal optical imaging, especially in the near infrared region (NIR), for human studies . ${ }^{12}$

The term of QD is generally supposed of spherical nanocrystals in the size ranges of $1-10 \mathrm{~nm}$ diameter.$^{13}$, but it can be produced in the other shapes such as rods and tetrapod.${ }^{14}$ however, the most widely used QDs for biological applications are spherical, and will be the focus of the current review article.

\section{Bulk semiconductor physics}

Based on electrical conductivity, solid state physics classifies materials into three categories: insulators, semiconductors, and conductors (Figure 1). The conductivity is generally expressed as the difference in energy between the valence and conduction bands. The valence band has the highest energy level which is occupied with electrons at room temperature. Likewise, the conduction is the lowest energy electronic state that may be occupied by thermal excitation. An electron in the valence band by gaining energy (by the absorption of a photon or thermally) can enter the conduction band and leave a positively charged hole in the valence band. In semiconductors, Band-gap energy (Eg) typically expressed as the difference in energy between the valence and conduction bands are small enough that some electrons could be thermally excited at room temperature to form charge carriers.$^{15}$

\section{Photo-physic of QDs}

An interesting characteristic of particulate semiconductors is that the Eg not only depends on the composition but are also affected by the particle size. Typically referred to as the "quantum confinement effect," this size dependency is remarkable when one or more dimensions of a semiconductor decrease to the nanometer scales. When all dimensions are confined to the nano-scaled ranges, the nanoparticle is named as a QD, which has characteristics between bulk state and individual atoms. The dependence of emitted wavelength on the size of nanoparticles make the development of new fluorescence 
nano-probes possible for obtaining tunable emission wavelengths. For example, in bulk state cadmium selenide (CdSe) has $\mathrm{Eg}$ of $1.7 \mathrm{eV}$ corresponding to $730 \mathrm{~nm}$ light emission, while QD of this material can be engineered to emit 450-650 nm by tuning the diameter of QDs from 2 to $7 \mathrm{~nm}$ (Figure 2). Additionally, the composition of the material is another important parameter which alters the Eg of a semiconductor. QD with a diameter of $5 \mathrm{~nm}$ can be engineered to radiate photon between $610-800 \mathrm{~nm}$ by changing the construction of the alloy CdSe and Te (Figure 2). ${ }^{16}$


Figure I Schematic diagrams showing band-gaps and charge carriers in bulk materials.

A: Solid state materials are classified as either electrical insulators, semiconductors or conductors, depending on the band-gap energy $(E$, between the valence and conduction bands. The valence bands are below the conduction bands for each material type, and electron occupation is represented by black shading.

B: In semiconductor, Absorption of a photon causes the excitation of an electron, generating charge carriers, an electron in the conduction band and a hole in the valence band. These two carriers quickly lose energy as they relax to the band edges, and their recombination leads to the emission of a photon.

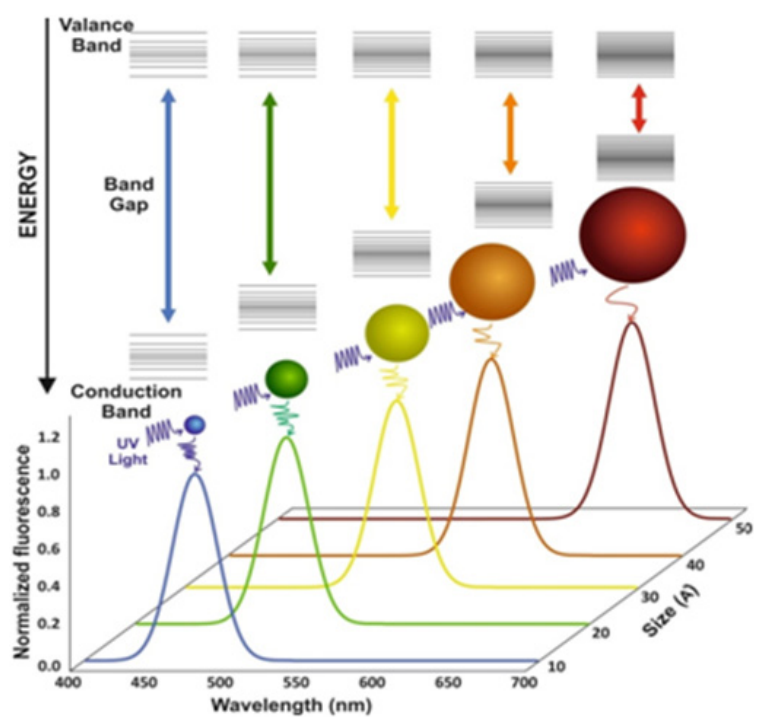

Figure 2 Size and composition tuning of optical emission for binary CdSe and ternary CdSeTe quantum dots. CdSe QD with various sizes (given as diameter) may be tuned to emit throughout the visible region by changing the nanoparticle size while keeping the composition constant. The size of QD may also be held constant, and the composition may be used to alter the emission wavelength.

\section{Comparison with organic dyes}

Organic fluorescent materials are extensively exploited as labeling dyes for fluorescence imaging. Due to the unique spectroscopic characteristics of QDs in comparison with organic dyes, QD opened new opportunities in a number of research areas. As shown in Figure 3, QD emit narrow/symmetric emission peaks ${ }^{17}$ (typically $25-35 \mathrm{~nm}$ full width at half maximum), which can be exited with a single light source due to the broad excitation profiles.
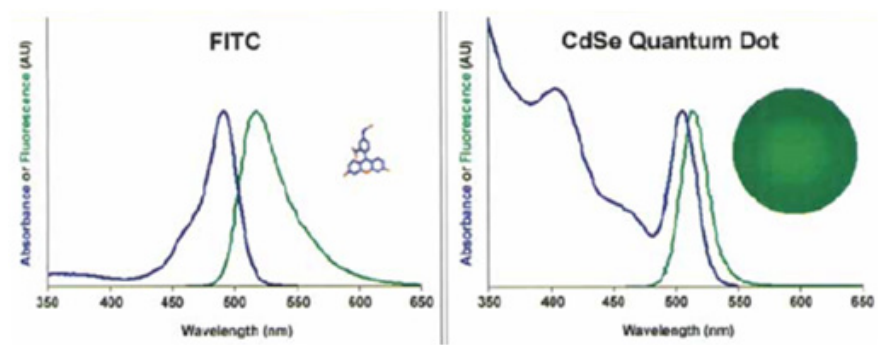

Figure 3 Comparison of absorbance (blue) and fluorescence (green) spectra between organic dyes (fluorescein isothiocyanate [FITC]) and CdSe quantum dots with the same emission wavelength. For the QD spectrum, the emission peak is more narrow and symmetric, and the absorption band extends far into the ultraviolet region. The size differences of FITC and QD are also shown ${ }^{15}$ (permission was obtained from John Wiley and Sons).

Therefore, multicolored QD can be simultaneously obtained with minimum optical overlapping, offering substantial advantages for multiplex analysis of molecular targets. Furthermore, QD seems to remain stable and bright after long episodes of excitation, whereas organic fluorophores are affected by photo-bleaching rapidly.${ }^{18}$ For these reasons, QD provide the possibility of real-time and continuous imaging single cells or single molecules over an extended period of time.

\section{Synthesis and bioconjugation}

\section{Synthesis and capping}

CdSe quantum dots (QDs) have received significant attention during the past decade due to their unique physicochemical properties .$^{19}$ So far, a number of methods such as the sonochemical method.$^{20}$ the microwave irradiation method.$^{21}$ and the organometallic precursor method.$^{22}$ have been developed for the preparation of CdSe QDs. The most popular technique for the synthesis of high quality CdSe QDs is the organometallic precursor method, however, due to the nonpolar nature of as-prepared QDs further process is needed for dispersing these QDs in water, necessary for use in biological system. It is very important issue that we would be able to develop some alternative methods for QDs synthesis in aqueous media. Biomedical applications basically require high-quality water-soluble QDs. Quantum dots could be prepared directly in aqueous media but often provide broader size distribution (leads to wide FWHM).$^{23}$ On the contrary, quantum dots obtained from organic solvent synthesis strategies, by simply changing the size, composition and/or structure, have very wide emission color ranging from ultraviolet to near infrared (300$2500 \mathrm{~nm}$ ) with reasonable monodispersity (leads to narrow FWHM) .${ }^{24}$ Hence, these quantum dots synthesized in organic solvents are insoluble in water and are challenging to make them soluble in water and also active in bioconjugation reactions. In this thesis, a novel and simple method reported to synthesize water soluble and small-size (1-3 nm) CdSe QDs is based on aqueous media synthesis which takes advantage from cysteine (cys) as stabilizing biocompatible agent. 


\section{Bioconjugation}

Most solubilizing agents used for QD synthesis in the aqueous media bear carboxylic acid groups release their protons in aqueous buffers and produce negatively charged colloids. Therefore, most strategies adapted to prepare bioconjugate QD rely on covalent bond formation between carboxylic acids and biomolecules such as proteins, nucleic acids and peptides. Moreover, since QD surface bear a net negative charge, positively charged molecules are electrostatically adsorbed on the surface of QDs, a method which has been exploited for the coverage of QD surface with cationic avidin proteins or recombinant maltose-binding proteins fused with positively charged peptides.$^{25}$ Alternatively, biomolecules containing basic functional groups such as thiols and amines moieties may also interact directly with QD surface as ligands.$^{26}$ If biomolecules not containing any groups for direct binding may be modified to introduce these functionalities; for example, peptides and nucleic acids can be modified with a thiol group for binding to QD.${ }^{27}$ Surface modification through high-affinity streptavidin-biotin binding is another approach which provides a convenient and indirect way for linking to a wide range of biotinylated biomolecules.$^{28}$ Zhang et al. ${ }^{29}$ conjugated streptavidin-modified quantum dots with biotin-derivatized aptamer via the interaction between biotin and streptavidin. They used this biocompatible bioprobe for imaging mouse liver hepatoma cell (MEAR cells) line.$^{29}$

\section{QDs as contrast agents for optical imaging}

Inorganic QDs are commonly classified into major two types depending on their chemical composition. In the first classification, QDs are composed of elements from groups III to V of the periodic table.$^{30}$ for example, Indium (In) from group III, phosphorus (P) from group V. In the second classification, QDs are composed of elements from groups II-VI of the periodic table; for example, cadmium (Cd) from group II, Selenium (Se) from group VI. The second category especially CdSe.$^{31}$ and CdTe.$^{32}$ are generally utilized in favor owing to their higher quantum yield compared to QDs from the first group. However, QDs from group 2 are potentially toxic because of the release of heavy metals, then, must be protected by engineering a physical shell between the main core and the environment. thioglycolic acid .${ }^{9}$ polyethylene glycol (PEG).$^{21}$ carboxyl- functionalized groups, as well as amine-functionalized groups.$^{33}$ are conventional barriers used in reducing QDs toxicities.


Figure 4 Detection of cancer markers on cell surfaces using immunoglobulinand monoclonal antibody-conjugated quantum dots. (A \& C) Detection of HER2- positive cancer cells using different sized (hence different colored) quantum dots. (B \& D) No significantly detectable fluorescence observed in HER2-negative cells ${ }^{18}$ (Permission was obtained from Nature Publishing Group).
These QDs also are used for real-time in vitro.$^{34}$ and in vivo. ${ }^{35}$ optical imaging of cancer cells. Cancer biomarkers such as prostatespecific antigen ${ }^{36}$ HER2 $2{ }^{37}$ CD $44 .{ }^{38}$ and folic acid ${ }^{39}$ specifically present in the tumor microenvironment or on the cancer cells surface, can be recognized by the appropriate monoclonal antibody ${ }^{40}$ peptide ${ }^{41}$ or immunoglobulin $.88,42$ that are conjugated onto the QDs surface (Figure 4). In some circumstances, it has been shown that QDs encapsulated in magnetic liposomes covered by Arginyl-GlycylAspartic acid (RGD) ligands have been utilized for the MRI imaging of tumor angiogenesis ${ }^{43}$

Apart from cancer imaging capability, QDs have also be utilized as 'light beacons' in which chemotherapeutic drugs such as doxorubicin (Dox) have interacted with QDs.$^{44}$ Once intracellularly delivering aptamer-functionalized QDs that were conjugated to Dox, upon the release of Dox from the nanosystem, the QDs fluorescent signals were on (Figure 5).

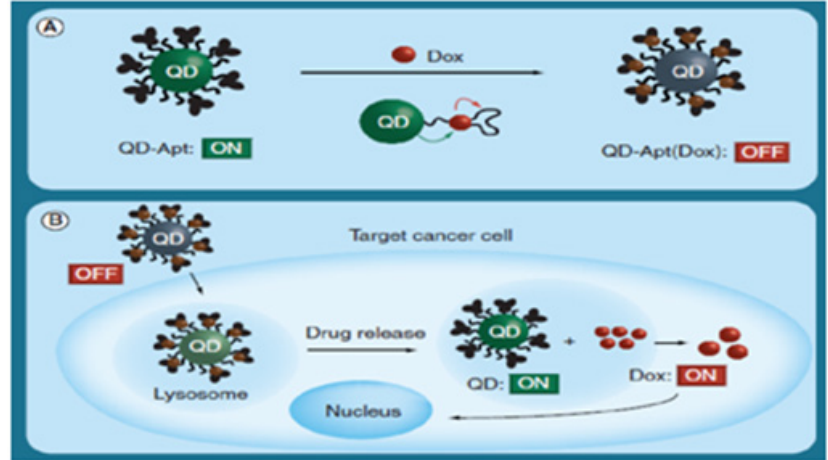

Figure 5 Quantum dots as 'light beacons' in chemotherapeutic drug delivery.

(A) Aptamer-functionalized QDs can be conjugated to Dox, which quenches the QD, rendering it none-fluorescent ('OFF' state).

(B) The release of Dox in the cell would render the QD in the 'ON' state, making it fluoresce ${ }^{44}$

(Permission was obtained from American Chemical Society).

Apt:Aptamer; Dox: Doxorubicin; QD: Quantum Dot

\section{Smart contrast agents}

As mentioned in the previous section, the fascinating optical characteristics of QDs make them even more appealing for molecular imaging. Currently, QDs commonly are conjugated with antibodies for the labeling of cancer cells or tissues that over-express specific cancer antigens ${ }^{45,46}$ Antibodies representing binding capacity to target antigens have been extensively utilized in the fields of cancer theranostic, but these methods have their own limitations including immunogenicity, incomplete target tissue penetration, long residence time in the blood stream, and being time consuming and costly in production process ${ }^{47,48}$ Therefore, exploiting other set of detecting molecules which show higher binding affinity and more accessibility by chemical synthesis to prepare conjugated quantum dots without aforementioned disadvantages would become a hotspot in the improvement of molecular probes ${ }^{49}$ Aptamers with similar characteristics to antibodies offering additional advantages of small size, easy synthesis and no immunogenicity ${ }^{50}$ have been successfully utilized in cancer imaging and cell labeling. For example, Chu et al. ${ }^{21}$ conjugated the aptamer of prostate-specific membrane antigen (PSMA) with QDs and evaluated the labeling of live cells, fixed cells, and prostate tumor cells (LNCaP) in a simulated tissue.$^{51}$ Chen et al. ${ }^{57}$ also attached a DNA aptamer of tenascin-C to QDs and accomplished the labeling of glioma cells expressing high levels of tenascin-C.$^{52}$ Moreover, other researchers conjugated both aptamer and peptide 
molecules with different colored QDs for simultaneous imaging of cancer cells. Ko and coworkers utilized dual color QDs attached to a AS1411 aptamer targeting nucleolin and a RGD tripeptide targeting the integrin $v 3$ for accomplishing the derby imaging of HeLa, MDAMB-231, and C6 cells . ${ }^{53}$ Hence, QDs conjugated to aptamers targeting cancer cells as real time fluorescent probes would provide promising prospects of application in the field of nanomedicine.

\section{Aptamers}

Aptamers are short artificial single-stranded DNA (ssDNA) or RNA oligonucleotides which bind to their targets with high selectivity and sensitivity due to the formation of three-dimensional structure.$^{54}$ By Andrew Ellington and co-workers, the word "aptamer" was derived from the Latin "aptus" (fitting) meant a nucleotide polymer that fits to its target ${ }^{38}$ Any class of substrate ranging from whole cells to small molecules such as peptides, drugs, and organic small molecules or even metal ions can be the target molecules for aptamers. Aptamers represent the potential applications in medical and pharmaceutical basic research including drug development, analytical science, diagnosis and therapy of disease owing to the specific and unique binding characteristics.$^{55}$ The technology to evolve aptamers was discovered in $1990.50,56$ and by Larry Gold was so called 'SELEX', Systematic Evolution of Ligands by Exponential enrichment.

\section{Structure and functionality}

The basic functionality of aptamers relies on stable threedimensional (3D) structure depending on the primary sequence, the environmental conditions and the length of the nucleic acid molecule (typically smaller than 100 nucleotides). The specific 3D structures of aptamers are identified by internal loops, stems, hairpins, bulges, tetra loops, triplicates, pseudo-knots, G-quadruplex structures, or kissing complexes. Binding of the aptamer to its target results from a combination of complementarity in the geometrical shape, stacking interactions of aromatic rings and the nucleobases of the aptamers, electrostatic interactions between charged groups, van der Waals interactions and hydrogen bonds.$^{57}$ Aptamers are named "nucleic acid antibodies" with additional superior characteristics ${ }^{58}$ Most aptamers display affinities comparable to those considered for monoclonal antibodies. Aptamers have capability to distinguish between chiral molecules, to recognize a distinct epitope of a target molecule and to differentiate between related targets, such as caffeine and theophylline. ${ }^{59}$

\section{SELEX process}

To make the SELEX technology more efficient and less time consuming, the selection process has frequently been modified for different applications and for different target molecules.$^{60}$ The initiation step of a common SELEX process is an artificially synthesized random DNA oligonucleotide library containing about 1013-1015 diverse sequences ${ }^{45}$ In the first round of SELEX process, the DNA or RNA pool is incubated with a specific target. Washing process separates weakly bound and unbound strands from target-oligonucleotide complexes. In the second step, the oligonucleotide sequences bound to target are amplified by reverse transcription PCR (RT-PCR) for RNA SELEX or by polymerase chain reaction (PCR) experiments for DNA SELEX. The resultant pool of enriched oligonucleotides would be used in the next round of SELEX procedure. Enrichment up to a saturation condition determines the end of SELEX process. The selected aptamer is cloned and sequenced for subsequent analysis and binding assays including the specificities and affinities.

\section{Advantages and properties of aptamers}

In comparison with antibodies, aptamers offer some advantages due to their unique physicochemical nature ${ }^{61}$

1. Aptamers especially DNA aptamers show higher stability, allowing convenient manipulation in harsh circumstances; they can be heated up to $95^{\circ} \mathrm{C}$ or exposed to organic solvents without showing considerable changes in functionality or structure. They also will return to their original conformation after recovery ${ }^{62}$

2. Easy chemical modifications are possible to immobilize them on surfaces or to extend their lifetime in biological fluids.

3. Whereas antibody-based imaging agents may circulate for days to weeks, aptamer-based imaging probes show short circulating half-lives in the body ${ }^{47}$

4. Sequences selected from initial SELEX procedure might be truncated for removing regions that are not important for direct interaction with the target. Sometimes such truncations even increase affinity of tailored aptamers.$^{63}$

Moreover, the smaller size of aptamers sometimes enable approach to epitopes that are inaccessible to antibodies ${ }^{64}$ Aptamers are utilized in conjugation with nanoparticles for sensing, imaging and smart drug delivery because of their potential capability by which nanosystem are bound to a targeted site, enabling active controlled delivery of drugs incorporated in the nanoparticulate.$^{58}$

\section{Cancer cell imaging by aptamer-conjugated QDs}

Tenascin-C is a dominant extracellular matrix protein that distributes on the surface of glioma cells. Chen et al. ${ }^{57}$ constructed a new bioprobe by conjugating GBI-10 aptamer to the QD surface, and used this fluorescent QD-labeled aptamer (QD-Apt) nanoprobe convenient and sensitive in vitro diagnostic assays to recognize the Tenascin-C molecules.$^{52}$ Likewise, Shieh et al. ${ }^{56}$ conjugated prostatespecific membrane antigen (PSMA) aptamers with QDs surface to label and image prostate carcinoma cells.

One of the major challenges of bioanalytical chemists is multiplex sensing of analytes specifically tumor markers ${ }^{4}$ Tried to image tenascin-C and nucleolin simultaneously using two types of aptamerconjugated QDs. they showed that this imaging was reliable and cell line-specific; however, current requirements for cancer imaging technologies include the need for simple preparation methods and the ability to detect multiple cancer biomarkers and evaluate their intracellular localizations ${ }^{65,66}$

Ko exploited AS1411 aptamer (targeting nucleolin) for engineering dual-colour QD based visualizing system to target biomarker with probes labeled with an aptamer and a peptide ${ }^{53}$ Simultaneous derby imaging of the cellular distribution of nucleolin and integrin using AS1411 aptamer modified QDs enables easy monitoring of separate targets in cancer cells and in normal healthy cells. The results suggested that synchronized visualization of cancer biomarkers would be possible via quantum dot-based aptameric nanosystem.

Detection of nucleolin protein expressed on extracellular matrix with AS1411 aptamer-conjugated CdTe QDs was another work that made targeted bio-imaging of glioblastoma cells (U87 cell line) possible.$^{67} \mathrm{Liu}$ and coworkers developed an effective strategy by which Mucin-1 (MUC-1) aptamer was covalently conjugated to magnetic beads for selective collection and detection of breast cancer cells ${ }^{68}$ Since MUC-1 antigen is over-expressed on epithelial cancer cells, the developed nano-probe containing aptamer was able to specifically detect captured cells. 
The MUC-1 is a protein that contains an extracellular domain consisting of 20 amino acids per repeat.$^{69}$ and a cytoplasmic domain of 69 amino acids.${ }^{70}$ MUC-1 function as a protecting element when pathogens bind to biological membrane.$^{71}$ and also as a signal transduction pathway.$^{72}$ In many cases, higher content of the MUC-1 protein, an extracellular glycoproteins.$^{70}$ that are bound to the cell surface via the formation of a gel matrix through transmembrane domains.$^{73}$ are found in blood samples of gastric, colorectal, prostate, breast, bladder, pancreatic, and lung carcinomas . ${ }^{74}$ This makes serum analysis for MUC-1 potentially valuable for tumor diagnosis, because almost all human epithelial adenocarcinomas express elevated levels of this protein on cell surfaces. At least, two types of assays based on MUC-1 antibody analyses have been proposed for monitoring its concentration: the CA 15-3 assay and the CA 27.29 assay.${ }^{75}$ Since large amounts of this protein are found in blood of cancer patients, serum analysis for MUC-1 can be potentially useful for early cancer detection. However, effective treatment of cancers deeply depends on the early detection of the malignancies.$^{76}$

Although two types of monoclonal antibody-based assays have been successfully developed to determine the concentration of MUC1 , the production of specific antibodies for these assays is a costly procedure and time-consuming. Additionally, the procedures need radioactive or fluorescent labeling of secondary antibodies. In this regards, an aptamer-based nanosystem for MUC-1 quantitation, with an ultimate goal of developing reliable and robust tools, will be useful for cancer imaging and therapy.

A DNA aptamer for MUC-1 antigen was first time selected by Ferreira et al. . ${ }^{77}$ The selected aptamers were shown to detect MCF-7 breast cancer cells. Cheng et al. ${ }^{57}$ introduced an aptameric detecting system based on a three-compartment DNA hybridization method for MUC-1 identification using quantum dot labeling. ${ }^{74}$ The sensor was based on a construct of three pre-determined DNA strands including the MUC-1 aptamer, a quencher sequence, and quantum dot-labelled reporter, which represent a strong fluorescence signal in the absence of the analyte. In the presence of MUC-1 antigen, the attenuated fluorescence signal is detected as a result of changes in the 3D structure aptamer strand upon binding to MUC-1. Since the fluorescence reporter and the quencher were brought into close proximity, FRET event (Förster resonance energy transfer or fluorescence resonance energy transfer) occurred between the QD and quencher. The LOD (limit of detection) for this analysis was at the nanomolar level. The method offered some advantages in the early diagnosis of epithelial cancers.

Shin et al. ${ }^{78}$ reported QD based probe (emission maxima at $565 \mathrm{~nm}$ ) covalently conjugated to MUC-1 aptamer. BOBO-3 that demonstrates significant fluorescence enhancement when intercalated into folded regions of aptamer with double helix structure was used to stain the duplex regions, resulting in the assembly of QD-aptamer conjugates over-loaded with $\mathrm{BOBO}-3$ molecules. In the absence of MUC-1 molecules, a FRET-associated emission of the intercalated BOBO-3 molecules is detected when QD is excited at $365 \mathrm{~nm}$. The excitation $(365 \mathrm{~nm})$ of aptamer conjugated QDs loaded with the intercalating dye molecules leads to the reduction of QD fluorescence intensity and the intensification of BOBO-3 fluorescence. On the other hand, the presence of MUC-1 molecules bound to the aptamer causes significant conformational change in folding of aptamer molecules, leading to the detachment of BOBO-3 molecules from aptamer conjugated QDs. Hence, a reduction in the FRET-associated BOBO-3 emission and an enhancement in the QD fluorescence intensity are remarkable, which allow development of well-established assay for MUC-1 quantification.
Very recently, ${ }^{79,80}$ aptameric optical probes for fluorescent imaging and rapid monitoring of circulating cancer cells ${ }^{79,80}$ Detection of epithelial cell adhesion molecule (EpCAM/muc1) overexpression directed to the cancer metastasis provides noninvasively critical information about CTC (circulating tumor cell) occurrence. Shie et al. ${ }^{56}$ also exploited graphene quantum dots (GQDs) and molybdenum disulfide (MoS2) nanosheets for construction of fluorescence turn-on aptameric biosensor for EpCAM detection. ${ }^{81}$ They used PEGylated GQDs as donor molecules and the sensing platform was realized by adsorption of PEGylated GQD labelled EpCAM aptamer onto MoS2 surface via van der Waals force. The fluorescence signal of GQD was quenched by MoS2 nanosheets via FRET mechanism. Specific affinity interaction between aptamer and EpCAM protein, In the presence of EpCAM protein, could detach EpCAM aptamer-labeled GQD from MoS2 nanosheets, resulting in the restoration of fluorescence intensity.

Hashemian et al. ${ }^{82}$ introduced aptasensor based on FRET using CdS QDs as a donor and polypyrrole (Ppy) as an acceptor for the analysis of adenosine in urine samples of lung cancer patients. The QDs were covalently bonded to anti-adenosine aptamer where its fluorescence was quenched by Ppy.

\section{Aptamer-QDs conjugates as theranostic agents}

Apart from the use of aptamer conjugated QDs as a sensing platform for bio-analysis or contrast agents for cancer imaging, this emerging nano-conjugates has also been applied in targeted drug delivery of cancers. The promising proposition of multifunctional nanoparticles for cancer diagnostics and therapeutics has inspired the development of theranostic approach for improved cancer therapy.${ }^{83}$ Chemotherapeutics can either covalently or noncovalently be loaded onto aptameric nanoparticles. Covalent attachment of therapeutic agents to aptamer conjugated QDs are generally achieved through standard thio-ester, amide bond, carboxyl ester formation or similar methods. For non-covalent modification of nano-conjugates, hydrophobic interaction or electrostatic adsorption schemes are often employed. Regardless of the kind of loading strategy, conjugated QDs are very appropriate for in-vivo applications due to their biocompatibility upon bioconjugation process. Moreover, the active targeted delivery of drugs into a cell seems to be a powerful technology with extensive applications in medicine, including the treatment of cancers ${ }^{84}$ For example, chemotherapy is an essential approach to cancer treatment, but the effectiveness of chemotherapy is commonly limited by the adverse effects of chemotherapeutics that are used in most therapeutic procedures ${ }^{85}$ Since conventional anticancer agents cannot differentiate between healthy and cancer cells, higher efficacy of anticancer agents is connected with higher toxicity to healthy cells and tissue ${ }^{86}$ Targeted cancer therapy is a useful approach to achieve lower toxicity, in which conventional anticancer agents are selectively delivered to cancer cells, so that the adverse effects are, reduced significantly ${ }^{87} \mathrm{~A}$ typical targeted drug delivery system generally consists of a targeting system such as aptamer conjugated QDs which can specifically bind to specific tumor markers abundantly expressed in cancer cells and a traditional anticancer agent. Recently, targeting molecules based on aptamers have become the new generation targeting agents ${ }^{88}$ As detecting elements, aptamers have perfect capability to deliver drugs or nanoparticles to target cells due to the higher affinity and specific binding capacity.$^{89}$ Owing to these characteristics, aptamers have been used as innovative targeting agents in targeted drug delivery of prostate cancer cells.$^{90}$ It is worth noting that the cancer marker employed in targeted therapy must be a membrane protein overexpressed in tumor cells compared to expression in normal cells. In 2007, Farokhzad and co-workers developed an intricate system based on using a FRET system QD- 
aptamer assembly to sense the PSMA for cancer imaging, therapy, and drug delivery of doxorubicin. ${ }^{91}$ Additionally, Minko and colleagues attempted to deliver doxorubicin to ovarian cancer cells by applying QDs nanoparticles modified with MUC-1 aptamers.${ }^{92}$ Doxorubicin was conjugated to QDs via a $\mathrm{pH}$-sensitive hydrazone bond in order to achieve stability of the nanosytem in the systemic circulating system and drug release in the acidic environment of cancer cells. Based on animal imaging studies performed on xenografts mouse model of human ovarian cancer, more MUC-1 aptamer conjugated QDs accumulated in the tumors when compared with unmodified QDs. Exvivo organ analysis confirmed higher uptake in the tumor and lower uptake in other organs. Hence, the obtained data revealed the high potential of targeted QDs in cancer treatment.

Although QD applications have been limited by their cytotoxicities .${ }^{12}$ there have been more attempts to explore new QDs, or to reduce their side effects for in real in vivo human imaging. However, research will continue to respond this demand. $\mathrm{Su}$ and co-workers prepared a novel MUC-1 aptamer--(CGA)7-conjugated CuInS2 QDs for cancer imaging or for sensing of daunorubicin (DNR) delivery to the targeted tumor cells. DNR is one of the most widely used anticancer agents which can inhibit the proliferation of cancer cells through intercalating into the DNA structure in cell nuclei. In order to achieve high drug loading efficiency, DNR intercalated into the double-stranded CG sequence of the MUC-QDs, which was used for synchronous sensing and targeting prostate cancer cells. Gao et al. ${ }^{91}$ synthesized water-soluble aptamer-Ag2S QDs water-soluble Ag2S near-infrared (NIR) fluorescent quantum dots (QDs) for specific cancer imaging and photothermal therapy (PTT). Evidences show that polymer-encapsulated QDs are nontoxic to cells and animals, but their long-term in vivo toxicity and degradation need more studies. Nevertheless, bioconjugated QDs have raised new possibilities for ultrasensitive and multiplexed imaging of molecular targets in living cells, animal models, and, possibly, in human patients. A new structural design represented was encapsulating luminescent QDs with amphiphilic block copolymers, and linking the polymer coating to tumor targeting ligands and drug-delivery functionalities. Active targeting of drug carrier to specific target site is crucial for providing efficient delivery of therapeutics and imaging agents. In this regard. Evaluated the theranostic capabilities of nutlin-3a loaded poly (lactide-co-glycolide) nanoparticles, functionalized with a targeting ligand (EpCAM aptamer) and an imaging agent (quantum dots) for cancer therapy and bioimaging. Were developed aptamer-conjugated graphene QDs/Porphyrin derivative theranostic agent for intracellular cancer-related microRNA detection and fluorescence -Guided photothermal/photodynamic synergetic therapy.$^{79,92}$

Another drawback of inorganic QDs are their low sensitivity for efficient tissue imaging. A Fluorescent Polymeric Quantum Dot/ aptamer superstructure and applied them for Imaging of Cancer Cells. In a way that first aptamer molecules and carboxylated QDs were conjugated with acrylamide monomers and then polymerized with amino persulfate (APS). After polymerization of aptamer, QDsconjugated polyacrylamide they used for imaging cancer cells with ultrasensitive staining.

\section{Summary}

In this Review, we discussed the use of QD-aptamer bioconjugates for cancer treatment and imaging in animal and in vitro studies. Fluorescent Semiconductor QDs are valuable tools for future theranostic investigations, because their stable fluorescent emission and capability of being modulated or conjugated with biocompartments such as enzymes, DNA, RNA, monoclonal antibodies suggest new opportunities. While bulk semiconductors do not have quantum confinement effect, nanoscaled inorganic semiconductors represents fascinating optical properties that introduce them as an efficient platform for cancer imaging and therapy; however, their toxicities restrict their uses in human studies.

\section{Conclusion}

In conclusion, we need to develop new safe QDs for human studies and to conjugate novel drugs or bio-compartments for obtaining a perfect theranostic agent.

\section{Acknowledgements}

The authors would like to acknowledge school of pharmacy at Ardabil University of Medical Sciences (ARUMS) for support.

\section{Conflict of interest}

None.

\section{References}

1. J Ferlay, I Soerjomataram, R Dikshit, S Eser et al. Cancer incidence and mortality worldwide: sources, methods and major patterns in GLOBOCAN 2012. Int J Cancer. 2014;136(5):E359-E386.

2. Frangioni JV New technologies for human cancer imaging. $J$ Clin Oncol. 2008;26(24):4012-4021.

3. P Karami, MR Majidi, M Johari Ahar et al. Development of screenprinted tryptophan-kynurenine immunosensor for in vitro assay of kynurenine-mediated immunosuppression effect of cancer cells on activated T-cells. Biosens and Bioelectron. 2016;92:287-293.

4. J Liu, JH Lee, Y Lu Quantum dot encoding of aptamer-linked nanostructures for one-pot simultaneous detection of multiple analytes. Anal Chem. 2007;79(11):4120-4125.

5. HS Choi, W Liu, F Liu et al. Design considerations for tumour-targeted nanoparticles. Nature nanotechnology. 2010;5(1):42-47.

6. Thiesen B, Jordan A Clinical applications of magnetic nanoparticles for hyperthermia. Int J Hyperthermia. 2008;24(6):467-474.

7. Robertson JH, Yang SY, Iga AM, et al.An in vivo rat model for early development of colorectal cancer metastasis to liver. Int J Exp Pathol. 2008;89(6):447-457.

8. Ghasemi Younes, Payam Peymani, S Saba Afifi Quantum dot: Magic nanoparticle for imaging, detection and targeting. Acta Biomed. 2009;80(2):156-165.

9. Zeenia Kaul, Tomoko Yaguchi, Sunil C Kaul et al. Mortalin imaging in normal and cancer cells with quantum dot immuno-conjugates. Cell Research. 2003;13:503-507.

10. Rile Li, Hong Dai, Thomas M Wheeler et al. Prognostic value of Akt-1 in human prostate cancer: A computerized quantitative assessment with quantum dot technology. Clinical Cancer Research. 2009;15(10):35683573 .

11. Olaf Minet, Cathrin Dressler, Jürgen Beuthan Heat Stress Induced Redistribution of Fluorescent Quantum Dots in Breast Tumor Cells. Journal of Fluorescence. 2004;14(3):241-247.

12. Saberian-Borujeni M, Johari-Ahar M, Hamzeiy H et al. Nanoscaled aptasensors for multi-analyte sensing. BioImpacts. 2014;4(4):205-215.

13. Michalet X, Pinaud FF, Bentolila LA et al. Quantum dots for live cells, in vivo imaging, and diagnostics. Science. 2005;307(5709):538-544.

14. X Peng Mechanisms for the shape-control and shape-evolution of colloidal semiconductor nanocrystals. Advanced Materials. $2003 ; 15(5): 459-463$. 
15. Andrew M Smith, Xiaohu Gao, Shuming Nie Quantum dot nanocrystals for in vivo molecular and cellular imaging. Photochemistry and photobiology. 2004;80(3):377-385.

16. Bailey RE, Nie S Alloyed semiconductor quantum dots: tuning the optical properties without changing the particle size. J Am Chem Soc. 2003;125(23):7100-7106

17. Gao X, Chan WC, Nie S Quantum-dot nanocrystals for ultrasensitive biological labeling and multicolor optical encoding. $J$ Biomed Opt. 2002;7(4):532-537.

18. Xingyong $\mathrm{Wu}$, Hongjian Liu, Jianquan Liu et al. Treadway, et al. Immunofluorescent labeling of cancer marker Her2 and other cellular targets with semiconductor quantum dots. Nat biotechnol. 2003;21(1):41-46.

19. Bentolila LA, Michalet X, Pinaud FF et al. Quantum dots for molecular imaging and cancer medicine. Discov Med. 2005;5 (26):213-218.

20. H Li, C Han Sonochemical Synthesis of Cyclodextrin-Coated Quantum Dots for Optical Detection of Pollutant Phenols in Water. Chemistry of Materials. 2008;20(19):6053-6059.

21. Maoquan Chu, Xin Song, Duo Cheng et al. Preparation of quantum dotcoated magnetic polystyrene nanospheres for cancer cell labelling and separation. Nanotechnology. 2006;17:13.

22. Mekis I, Talapin DV, Kornowski A et al. One-pot synthesis of highly luminescent $\mathrm{CdSe} / \mathrm{CdS}$ core-shell nanocrystals via organometallic and "greener" chemical approaches Journal of Physical Chemistry B. 2003;107:7454-7462.

23. J Zhuang, X Zhang, G Wang et al. Synthesis of water-soluble ZnS: $\mathrm{Mn} 2+$ nanocrystals by using mercaptopropionic acid as stabilizer. $J$ Mater Chem. 2003;13(7):1853.

24. C Murray, DJ Norris, MG Bawendi Synthesis and characterization of nearly monodisperse $\mathrm{CdE}$ ( $\mathrm{E}=$ sulfur, selenium, tellurium) semiconductor nanocrystallites. Journal of the American Chemical Society. 1993;115(19):8706-8715.

25. H Mattoussi, JM Mauro, ER Goldman et al. Self-Assembly of CdSe-ZnS Quantum Dot Bioconjugates Using an Engineered Recombinant Protein. Journal of the American Chemical Society. 2000;122(49):12142-12150.

26. X Gao, WC Chan, S Nie Quantum-dot nanocrystals for ultrasensitive biological labeling and multicolor optical encoding. J Biomed Opt. 2002; 7(4):532-537.

27. GP Mitchell, CA Mirkin, RL Letsinger Programmed Assembly of DNA Functionalized Quantum Dots. Journal of the American Chemical Society. 1999;121(35):8122-8123.

28. X Wu, H Liu, J Liu, KN Haley et al. Immunofluorescent labeling of cancer marker Her2 and other cellular targets with semiconductor quantum dots. Nature biotechnology. 2003;21:41-46.

29. J Zhang, X Jia, XJ Lv et al. Fluorescent quantum dot-labeled aptame bioprobes specifically targeting mouse liver cancer cells. Talanta. 2010;81(1-2):505-509.

30. ES Glazer, SA Curley Radiofrequency field-induced therma cytotoxicity in cancer cells treated with fluorescent nanoparticles. Cancer. 2010;116(13):3285-3293.

31. H Kobayashi, M Ogawa, N Kosaka et al. Polychromatic in vivo imaging of multiple targets using visible and near infrared light. Adv Drug Deliv Rev. 2009;65(8):1112-1119.

32. P Diagaradjane, JM Orenstein-Cardona, NE Colon-Casasnovas et al. Imaging epidermal growth factor receptor expression in vivo: pharmacokinetic and biodistribution characterization of a bioconjugated quantum dot nanoprobe. Clin Cancer Res. 2008;14(3):731-741.

33. A Ghazani, JA Lee, J Klostranec et al. High throughput quantification of protein expression of cancer antigens in tissue microarray using quantum dot nanocrystals. Nano Lett. 2006;6(12):2881-2886.
34. A Zajac, D Song, W Qian, T Zhukov Protein microarrays and quantum dot probes for early cancer detection. Colloids Surf B Biointerfaces. 2007;58(2):309-314.

35. TA Zdobnova, SG Dorofeev, PN Tananaev et al. Quantum dots for molecular diagnostics of tumors. Biochem Biophys. 2010;430:441.

36. Johari Ahar M, J Barar, AM Alizadeh et al. Methotrexate-conjugated quantum dots: synthesis, characterisation and cytotoxicity in drug resistant cancer cells. J Drug Target. 2016;24(2):120-133.

37. SM Farkhani, M Johari ahar, P Zakeri-Milani et al. Drug delivery and nanodetection in lung cancer. Artif Cells Nanomed Biotechnol. 2016;44(2):618-634.

38. R Smith, Z Cheng, A De, AL Koh et al. Real-time intravital imaging of RGD-quantum dot binding to luminal endothelium in mouse tumor neovasculature. Nano Lett. 2008;8(9):2599-2606.

39. X Yu, L Chen, K Li et al. Immunofluorescence detection with quantum dot bioconjugates for hepatoma in vivo. J Biomed Opt. 2007;12:014008.

40. A Gokarna, LH Jin, JS Hwang et al. Quantum dot-based protein microand nanoarrays for detection of prostate cancer biomarkers. Proteomics. 2008;8(9):1809-1818.

41. H Tada, H Higuchi, TM Wanatabe, N Ohuchi In vivo real-time tracking of single quantum dots conjugated with monoclonal anti-HER 2 antibody in tumors of mice. Cancer Res. 2007;67(3):1138-1144.

42. EL Snyder, D Bailey, M Shipitsin, et al. dentification of CD44v6(+)/ CD24- breast carcinoma cells in primary human tumors by quantum dot-conjugated antibodies. Lab Invest. 2009;89(8):857-866.

43. KT Yong, I Roy, R Hu, H Ding, H Cai, et al. Synthesis of ternary CuInS2/ $\mathrm{ZnS}$ quantum dot bioconjugates and their applications for targeted cancer bioimaging Integr Biol (Camb). 2010;2:121-129.

44. MK Sadigh, M Zakerhamidi, SS Ahmadian et Al. Environment effect on spectral and charge distribution characteristics of some drugs of folate derivatives. Spectrochimica Acta Part A: Molecular and Biomolecular Spectroscopy. 2017;171:10-17.

45. H Zhang, X Zeng, Q Li, M Gaillard-Kelly et al. Fluorescent tumour imaging of type I IGF receptor in vivo: comparison of antibodyconjugated quantum dots and small-molecule fluorophore. British journal of cancer. 2009;101:71-79

46. Stroh M, Zimmer JP, Duda DG et al. Quantum dots spectrally distinguish multiple species within the tumor milieu in vivo. Nat Med. 2005;11(6):678-682

47. M Hu, J Yan, Y He, H Lu et al. Ultrasensitive, Multiplexed Detection of Cancer Biomarkers Directly in Serum by Using a Quantum Dot-Based Microfluidic Protein Chip. ACS nano. 2009;4(1):488-494.

48. WJ Mulder, K Castermans, JR Van Beijnum et al. Molecular imaging of tumor angiogenesis using alphavbeta3-integrin targeted multimodal quantum dots. Angiogenesis. 2009;12(1):17-24.

49. Bagalkot V, Zhang L, Levy-Nissenbaum E et al. Quantum Dot-Aptamer Conjugates for Synchronous Cancer Imaging, Therapy, and Sensing of Drug Delivery Based on Bi-Fluorescence Resonance Energy Transfer Nano Letters. 2007;7(10):3065-3075

50. Lee J, Choi Y, Kim K, Hong S et al. Characterization and Cancer Cell Specific Binding Properties of Anti-EGFR Antibody Conjugated Quantum Dots. Bioconjugate Chemistry. 2010;21(5):940-946

51. D Tiwari, SI Tanaka, Y Inouye et al. Synthesis and Characterization of Anti-HER2 Antibody Conjugated CdSe/CdZnS Quantum Dots for Fluorescence Imaging of Breast Cancer Cells. Sensors (Basel). 2009;9(11):9332-9364.

52. SD Jayasena Aptamers: an emerging class of molecules that riva antibodies in diagnostics. Clin Chem. 1999;45(9):1628-1650. 
53. JO Lee, HM So, EK Jeon et al. Aptamers as molecular recognition elements for electrical nanobiosensors. Analytical and bioanalytical chemistry. 2008;390(4):1023-1032.

54. Farokhzad OC, Jon S, Khademhosseini A et al. Nanoparticle-aptamer bioconjugates: a new approach for targeting prostate cancer cells. Cancer research. 2004;64(21):7668-7672.

55. C Tuerk, L Gold Systematic evolution of ligands by exponential enrichment: RNA ligands to bacteriophage T4 DNA polymerase. Science. 1990;249(4968):505-510.

56. TC Chu, F Shieh, LA Lavery et al. Aptamer mediated siRNA delivery. Nucleic acids research. 2006;34(10): e73.

57. XC Chen, YL Deng, Y Lin, DW Pang et al. Quantum dot-labeled aptamer nanoprobes specifically targeting glioma cells. Nanotechnology. 2008;19(23):235105.

58. Ebrahimi M, Johari Ahar M, Hamzeiy $H$ et al. Electrochemical impedance spectroscopic sensing of methamphetamine by a specific aptamer. Bioimpacts. 2012;2(2):91-95.

59. Molecular Imaging and Contrast Agent Database (MICAD). National Center for Biotechnology Information (US), Bethesda, USA.

60. AD Ellington, JW Szostak In vitro selection of RNA molecules that bind specific ligands. Nature. 1990;346(6287):818-822.

61. T Hermann, DJ Patel Adaptive recognition by nucleic acid aptamers. Science. 2000;287(5454):820-825.

62. VC Ozalp, F Eyidogan, HA Oktem Aptamer-Gated Nanoparticles for Smart Drug Delivery. Pharmaceuticals. 2011;4(3):1137-1157.

63. RD Jenison, SC Gill, A Pardi, B Polisky High-Resolution Molecular Discrimination by RNA. Science. 1994;263:1425-1429.

64. R Stoltenburg, C Reinemann, B Strehlitz SELEX - a (r)evolutionary method to generate high-affinity nucleic acid ligands. Biomol Eng. 2007;24(4):381-403.

65. MR Majidi, Y Omidi, P Karami et al. Reusable potentiometric screenprinted sensor and label-free aptasensor with pseudo-reference electrode for determination of tryptophan in the presence of tyrosine. Talanta. 2016;150:425-433.

66. DH Burke, L Scates, K Andrews et al. RNA aptamers to the peptidyl transferase inhibitor chloramphenicol. Chem Biol. 1997;4(11):833-843.

67. JF Lee, GM Stovall, AD Ellington Aptamer therapeutics advance. Current opinion in chemical biology. 2006;10(3):282-289.

68. Felice Shieh, Laura Lavery, Chitai Ted Chu et al. Semiconductor nanocrystal-aptamer bioconjugate probes for specific prostate carcinoma cell targeting. Progress in Biomedical Optics and Imaging Proceedings of SPIE. 2005;36:159-165.

69. J Lee, HJ Kang, H Jang et al. Simultaneous imaging of two different cancer biomarkers using aptamer-conjugated quantum dots. Sensors (Basel). 2015;15(4):8595-8604.

70. M Alibolandi, K Abnous, M Ramezani et al. Synthesis of AS1411aptamer-conjugated CdTe quantum dots with high fluorescence strength for probe labeling tumor cells. J Fluoresc. 2014;24(5):1519-1529.

71. X Hua, Z Zhou, L Yuan, S Liu Selective collection and detection of $\mathrm{MCF}-7$ breast cancer cells using aptamer-functionalized magnetic beads and quantum dots based nano-bio-probes. Anal Chim Acta. 2013;788:135-140.

72. S Müller, K Alving, J Peter-Katalinic et al. High density O-glycosylation on tandem repeat peptide from secretory MUC1 of T47D breast cancer cells. J Biol Chem. 1999;274(26):18165-18172.

73. SJ Gendler, AP Spicer Epithelial mucin genes. Annual Review of Physiology. 1995;57:607-634.
74. McAuley JL, Linden SK, Chin WP et al. MUC1 cell surface mucin is a critical element of the mucosal barrier to infection. Journal of Clinical Investigation. 2004;117(8):2313-2324.

75. Hollingsworth M A, Swanson B J Mucins in cancer: protection and control of the cell surface. Nature Reviews Cancer. 2004;4:45-60.

76. Xueqin Wang, Li Ren, Qiang Wang et al. Conjugation of Biomolecules with Magnetic Protein Microspheres for the Assay of Early Biomarkers Associated with Acute Myocardial Infarction. Analytical Chemistry. 2009;81(15):6210-6217.

77. Gijsbert G Bon, Silvia von Mensdorff-Pouilly, Peter Kenemans, Gerard J van Kamp, Rob A Verstraeten, et al. Clinical and technical evaluation of ACSTM BR serum assay of MUC1 gene-derived glycoprotein in breast cancer, and comparison with CA 15-3 assays. Clinical Chemistry. 1997;43(4):585-593.

78. Croce MV, Isla-Larrain MT, Demichelis SO et al. Tissue and Serum MUC1 Mucin Detection in Breast Cancer Patients. Breast Cancer Research and Treatment. 2003;81(3):195-207

79. Johari-Ahar M, Rashidi M R, Barar J et al. An ultra-sensitive impedimetric immunosensor for detection of the serum oncomarker CA-125 in ovarian cancer patients. Nanoscale. 2015;7:3768-3779.

80. CSM Ferreira, CS Matthews, S Missailidis DNA aptamers that bind to MUC1 tumour marker: design and characterization of MUC1-binding single-stranded DNA aptamers. Tumor Biology. 2006;27(6):289-301.

81. Z Hashemian, T Khayamian, M Saraji et al. Aptasensor based on fluorescence resonance energy transfer for the analysis of adenosine in urine samples of lung cancer patients. Biosens Bioelectron. 2016;79:334-340.

82. J Barar, V Kafil, MH Majd et al. Multifunctional mitoxantroneconjugated magnetic nanosystem for targeted therapy of folate receptoroverexpressing malignant cells. Nanobiotechnology. 2015;13:26.

83. V Bagalkot, OC Farokhzad, R Langer, S Jon An aptamer-doxorubicin physical conjugate as a novel targeted drug-delivery platform. Angew Chem Int Ed Engl. 2006;45(48):8149-8152.

84. TA Yap, CP Carden, SB Kaye Beyond chemotherapy: targeted therapies in ovarian cancer. Nat Rev Cancer. 2009;9(3):167-181.

85. I Brigger, C Dubernet, P Couvreur Nanoparticles in cancer therapy and diagnosis. Adv Drug Deliv Rev. 2002;54(5):631-651.

86. E Levy Nissenbaum, AF Radovic Moreno, AZ Wang, R Langer et al. Nanotechnology and aptamers: applications in drug delivery. Trends Biotechnol. 2008;26(8):442-449.

87. J Zhou, JJ Rossi Development of cell-type specific anti-HIV gp120 aptamers for siRNA delivery. $J$ Vis Exp. 2011 ;(52):2954.

88. S Dhar, FX Gu, R Langer Targeted delivery of cisplatin to prostate cancer cells by aptamer functionalized Pt (IV) prodrug-PLGA-PEG nanoparticles. Proc Natl Acad Sci USA. 2008;105(45):17356-17361.

89. MS Zakerhamidi, M Johari-Ahar, SM Seyed Ahmadian et al. Photophysical behavior of some antitumor anthracycline in solvent media with different polarity. Spectrochimica Acta Part A: Molecular and Biomolecular Spectroscopy. 2014;130:257-262.

90. Z Lin, Q Ma, X Fei, H Zhang, X Su A novel aptamer functionalized CuInS2 quantum dots probe for daunorubicin sensing and near infrared imaging of prostate cancer cells. Anal Chim Acta. 2014;818:854-860.

91. X Gao, Y Xing, LWK Chung, S Nie In vivo molecular and cellular imaging with quantum dots. Curr Opin Biotechnol. 2005;16(1):63-72.

92. M Das, W Duan, SK Sahoo Multifunctional nanoparticle-EpCAM aptamer bioconjugates: a paradigm for targeted drug delivery and imaging in cancer therapy. Nanomedicine. 2015;11(2):379-389. 